\title{
Towards prediction of cake detachment in tailings filtration
}

\author{
B Fränkle Karlsruhe Institute of Technology, Germany \\ P Morsch FLSmidth \& Co. A/S, Denmark, and Karlsruhe Institute of Technology, Germany \\ H Nirschl Karlsruhe Institute of Technology, Germany
}

\begin{abstract}
By offering an opportunity to fulfil environmental and process water recovery specifications, filtration of tailings has received increasing attention. In the process, complete cake detachment is required to maintain high throughputs. Incomplete discharge represents a bottleneck in the process. Therefore, a deeper understanding of cake detachment behaviour is a key to success. Experimental studies on adhesion forces between filter cake and cloth are a first step towards detachment prediction. Filtrations using an FLSmidth lab filter press and subsequent adhesion measurements with iron ore tailings and two different filter media show the influence of filtration pressure on adhesion.
\end{abstract}

Keywords: tailings filtration, filter press, adhesion, cake detachment

\section{Introduction}

Demand for commodities is in a sustained upward trend. As a result, the amount of mined rock and associated tailings to be handled is increasing (FLSmidth 2020). The method of dry stacked tailings is becoming increasingly established to enable safe storage of these tailings (Davies 2011). Furthermore, a high amount of process water can be recovered, reducing operational costs of tailings processing.

However, the filtration of tailings is not a trivial matter. In addition to the technical process and equipment involved, which is associated with investment and operating costs, a solid-liquid separation task must be solved that requires a certain amount of background knowledge. In the process, the rock has to be crushed until the individual particles consist of only one material, so that the valuable material can be separated. For this reason, tailings usually have a particle size distribution in the range below $100 \mu \mathrm{m}$ with a simultaneously high clay content (Wang et al. 2014). Since clay material has a compressible behaviour this has an influence on the cake build-up and, therefore, on the filtration process (Wakeman 2007). High driving forces are required to achieve the target residual moistures of 20 mass percent or less. Filter presses can filter with high pressure differences and are therefore found in this application. Due to the large amount of tailings to be processed, correspondingly large presses are necessary, of which, moreover, several are operated in parallel arrangement (Wisdom et al. 2020). In addition to fabric blinding (Fränkle et al. 2021) and mechanical wear, cake sticking after filtration is a major problem that can occur during operation.

\section{Theory}

This section gives an overview of the basics of the filtration that takes place in the presses and the basics of the prerequisites of cake detachment.

\subsection{Wet cake filtration}

The underlying mechanism of filtration in filter presses is wet cake filtration. This is shown schematically in Figure 1. The suspension flows in the direction of a filter medium due to the differential pressure. This can be, for example, a metal or plastic mesh, or a flow. The aim is to separate the solid matter from the filter medium. The individual particles in the suspension are smaller than the pores of the medium, but if the concentration of particles in the solution is sufficient, individual particles only pass through into the filtrate 
at the beginning. The concentration of the particles is so high that bridging (i.e. mutual blocking of the particles above the pores) quickly occurs. Subsequently, arriving particles are deposited on the particulate network itself. The cake now grows steadily. There is usually an additional mechanical support structure behind the filter medium, which allows the filtrate to drain and ensures mechanical stability. This can also be incorporated into the fabric itself.

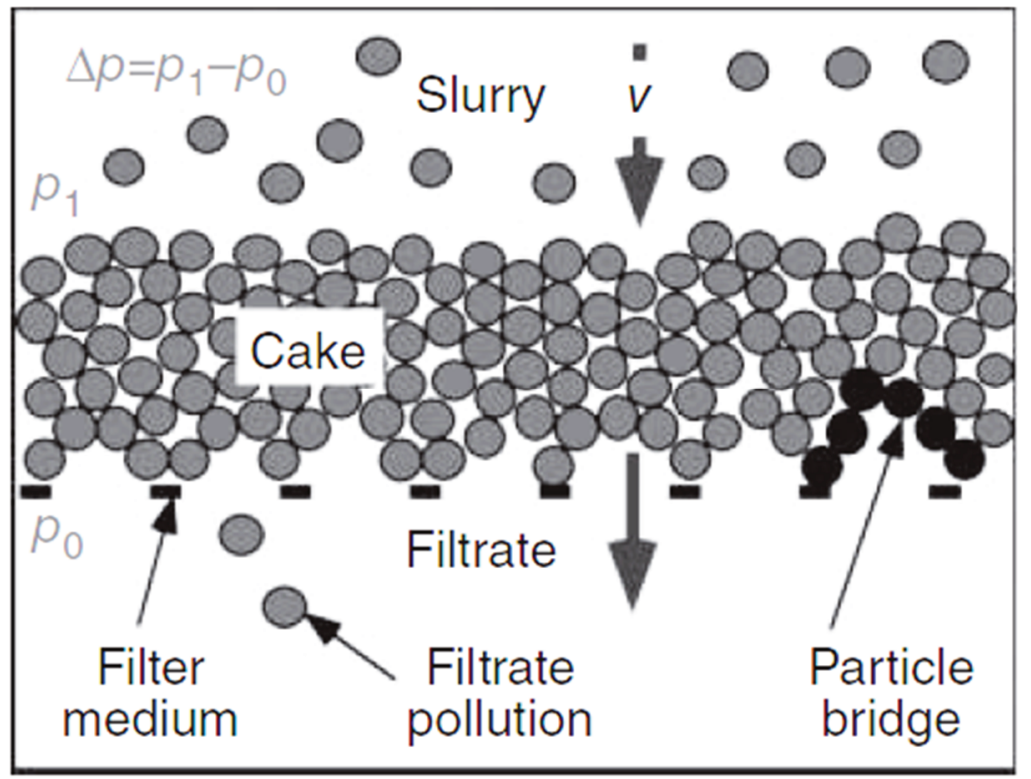

Figure 1 Schematic representation of the wet cake filtration process (Anlauf 2019)

Due to the growing cake, which is a porous structure, the pressure loss also increases. The Darcy equation (Darcy 1856) (Equation 1) describes this pressure loss and therefore is of technical relevance for the design of filtration apparatus (Ripperger et al. 2013). The empirical equation described by the French engineer in 1856 relates the flow rate $\dot{m}$ for a particulate network to the fluid density $\rho_{\text {Fluid, }}$, the dynamic viscosity of the fluid $\eta_{\text {Fluid, }}$ the height of the cake $h_{\text {cake, }}$, the area flowed through $A$, the pressure difference $\Delta p$, and the flowability P. Laminar flow conditions are assumed and friction is neglected.

$$
\frac{\dot{m}}{\rho_{\text {Fluid }} \cdot A}=P \cdot \frac{\Delta p}{\eta_{\text {Fluid }} \cdot h_{\text {Cake }}}
$$

The term of permeability is related to particulate network properties. Reciprocally, it corresponds to the product $\alpha_{H}$ of height-specific resistance and height. The equation of Kozeny and Carman (Equation 2 ) is a commonly used approximation of $\alpha_{H}$ (Kozeny 1927). Using the hydraulic diameter of a spherical particles system, the following relationship involving the Sauter diameter $d_{s}$ and porosity $\varepsilon$ can be stated (Ripperger et al. 2013).

$$
\alpha_{H} \approx 180 \cdot \frac{(1-\varepsilon)^{2}}{\varepsilon^{3} \cdot d_{S}^{2}}
$$

A reduction of particle diameter has a strong negative effect on permeability. Literature classifies the product of $\alpha_{H} \cdot \eta_{\text {Fluid }}$ in the range between very well filterable to almost unfilterable $\left(10^{11} \mathrm{mPa} \cdot \mathrm{s} \cdot \mathrm{m}^{-2}\right.$ to $\left.10^{16} \mathrm{mPa} \cdot \mathrm{s} \cdot \mathrm{m}^{-2}\right)$ (Rippberger et al. 2013).

A high clay content of tailings increases cake resistance. Furthermore, real-world solid-liquid separation tasks like tailings filtration have to deal with more complex influences of non-spherical and non-monodisperse particles. The size range of the clay content is in a range causing compressible behaviour, for example (Wakeman 2007). In order to ensure compaction of the particulate network and achieve crucial residual moisture contents, sufficient filtration pressure is necessary. Therefore, filter presses can be used (Gomes et al. 2016). 


\subsection{Cake detachment behaviour}

Ideally, when operating a filter press, the filter cakes of the individual chambers should detach from the filter medium in one piece when the respective chamber is opened and fall. Unfortunately, this is not always the case in real operation. In order to enable the detachment of the filter cake, it is absolutely necessary that the adhesion of the cake to the filter fabric is overcome by the cake's own weight and its ability to stay consolidated as a cake. Equation 3 represents this necessary condition.

$$
F_{\text {Gravitation }}>F_{\text {Adhesion }}
$$

Also necessary are statements about the behaviour of the cake itself. Weigert describes as a sufficient condition for successful cake dropping that the cohesion of the cake must be sufficiently high (Weigert 2001). This is shown schematically in Figure 2. The interaction between different materials (e.g. filter cake on the filter fabric) is called adhesion (red area). In the particulate network, it's called cohesion (green area). These are terms describing results of particle-wall interactions (red arrows) and particle-particle interactions (green arrows) in a macroscopic point of view.

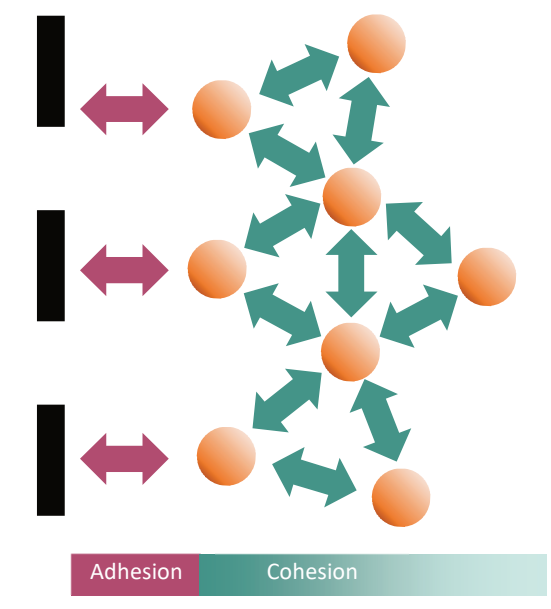

Figure 2 Schematic representation of adhesion and cohesion

Crucial here is a reference to the compressible behaviour already mentioned. In the filtration of tailings, the cake becomes increasingly compacted when filtration is carried out at a higher pressure. This leads to an increase in the contact points of the particles both on the fabric and in the cake, and thus to an increase in the transferable forces.

\section{$3 \quad$ Materials and methods}

A laboratory filter press from FLSmidth (Figure 3 ) is used to carry out filtrations at different pressures (2.5 bar and 12.5 bar). The slurry is filled into the cylindric inlet (inner diameter $10 \mathrm{~cm}$, height $4 \mathrm{~cm}$ ) having between the two endplates of the filter press using an inlet on the top of the cylinder. The filling process is carried out by the overlaying of a stirred pressure vessel with compressed air in which a riser pipe is located. The filtrate is gravimetrical measured using a laboratory scale. To reduce manual effort, the filter press is supported by a process control system allowing adjustment of filtration pressure, recording of filtrate amount, etc.

After filtration and opening of the filter press, the cylinder including the filter cake and the adhering cloths can be taken out and placed in a modified tensile testing machine. There the cloths can be clamped and pulled off. Thus, the shear force necessary to overcome adhesion can be determined. The pulling velocity is $10 \mathrm{~mm} \cdot \mathrm{min}^{-1}$.

Iron ore tailings were investigated, which were already part of investigation of filter media regeneration (Fränkle et al. 2021). These show a particle size distribution and mass concentration typical for tailings, which is listed in Table 1. 


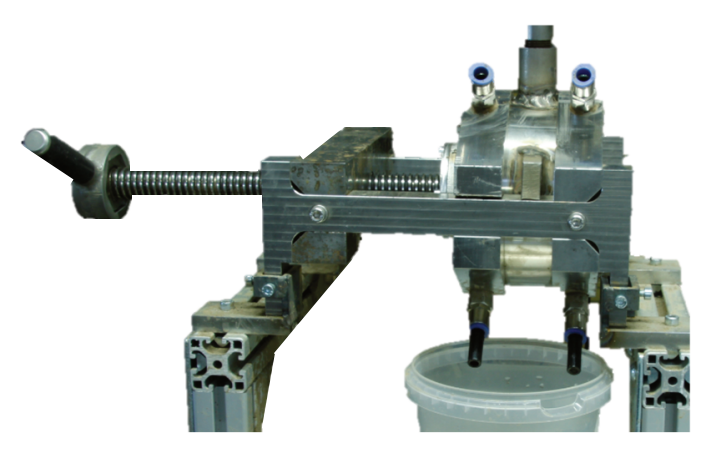

Figure 3 FLSmidth laboratory filter press

Table 1 Properties of the iron ore tailings (Fränkle et al. 2021)

\begin{tabular}{lll}
\hline Iron ore tailings & \\
\hline Solid density & & $3,050 \mathrm{~kg} \cdot \mathrm{m}^{-3}$ \\
Slurry concentration & $60 \mathrm{w}-\%$ \\
Particle size distribution & $\mathrm{x}_{10,3}$ & $7.2 \mu \mathrm{m}$ \\
& $\mathrm{x}_{50,3}$ & $38.2 \mu \mathrm{m}$ \\
& $\mathrm{x}_{90,3}$ & $120.8 \mu \mathrm{m}$ \\
\hline
\end{tabular}

Filtration tests were carried out with two filter media types typical for the application. A nylon cloth and polypropylene felt media were compared. Their properties are listed in Table 2. Filter media resistance measurements were carried out in a pressurised filter cell according to VDI Guideline 2762 (Verein Deutscher Ingenieure 2010). Mean flow pore was determined using a capillary flow porometer CFP 1500 AFX (Porous Materials Inc.) and a linear, non-reactive polydimethylsiloxane AK 10 (Wacker Chemie AG) as wetting agent.

Table 2 Properties of the two filter media

\begin{tabular}{lll}
\hline & PA-cloth & PP-felt \\
\hline Image & \multicolumn{1}{c}{ Plain } & \\
& Mono/mono & - \\
Weave-type & $240 \pm 20 / 250 \pm 20 \mu \mathrm{m}$ & - \\
Fibre type & $9.8 \pm 0.3 \cdot 10^{7} \mathrm{~m}^{-1}$ & $9.4 \pm 1.9 \cdot 10^{8} \mathrm{~m}^{-1}$ \\
Fibre diameter (warp/weft) & $1.5 \pm 0.1 \mathrm{~mm}$ \\
Resistance & $1.2 \pm 0.1 \mathrm{~mm}$ & $20 \pm 2 \mu \mathrm{m}$ \\
Thickness & $72 \pm 3 \mu \mathrm{m}$ & - \\
Mean flow pore & &
\end{tabular}

\section{$4 \quad$ Results}

In Figure 4, the measured adhesions of the different fabrics are shown for each filtration pressure. It can be seen that a higher filtration pressure, and thus a higher compaction of the cake, increases adhesion. This is particularly pronounced for the felt filter medium. Furthermore, higher adhesion values were measured for 
the felt in comparison to the nylon cloth. This is slightly pronounced at 2.5 bar and clearly visible at 12.5 bar. A higher adhesion behaves linearly with the thickness of the cake, which would be necessary for a detachment.

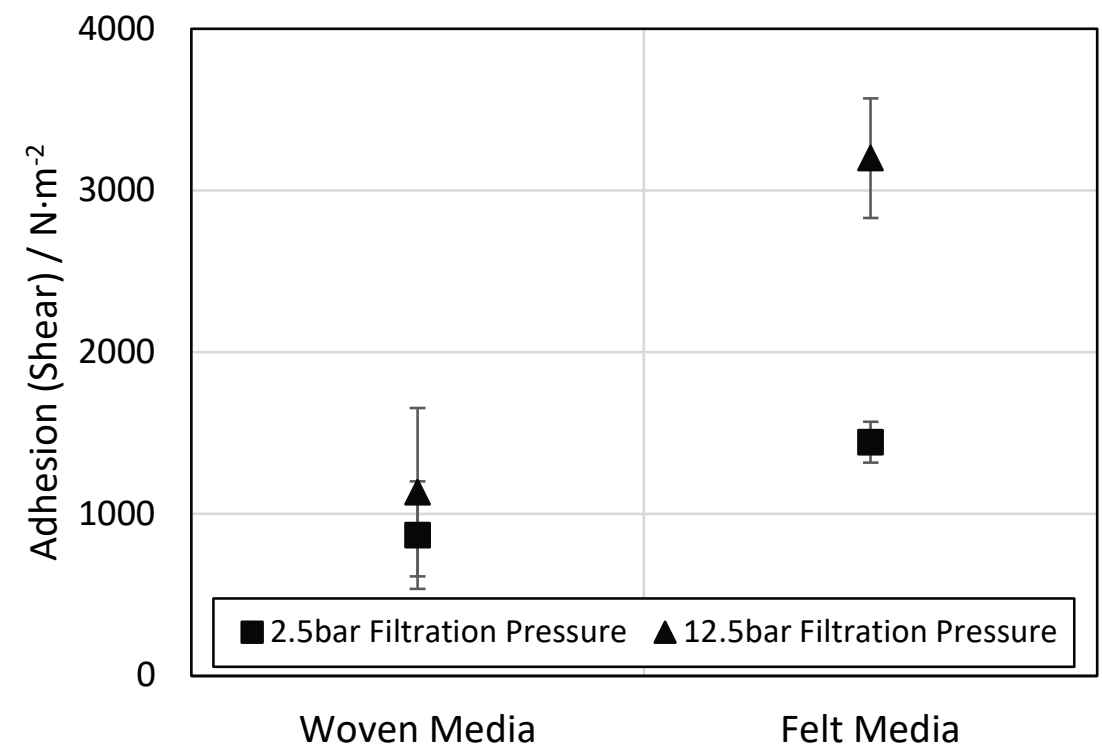

\section{Figure 4 Adhesion (shear) of the two filter fabrics for different filtration pressures}

Often the residual moisture of the cake is taken as the key parameter to describe filtration performance and cake detachment. It is used to characterise the throughput of the filter press, to derive the stability of the stacked material, but also to make statements about the detachment behaviour. The latter is often presented with experience from pure observation, but not with force measurements. For this reason, the measured values of the adhesion are plotted in Figure 5 via their associated residual moisture. The cake moistures are in a comparable range, but it can be stated that for each cloth a higher filtration pressure leads to a more compressed cake and therefore a lower moisture content, as expected. Adhesion also increases with decreasing residual moisture. This is also due to a stronger compression of the cake, which leads to an increased number of contact points.

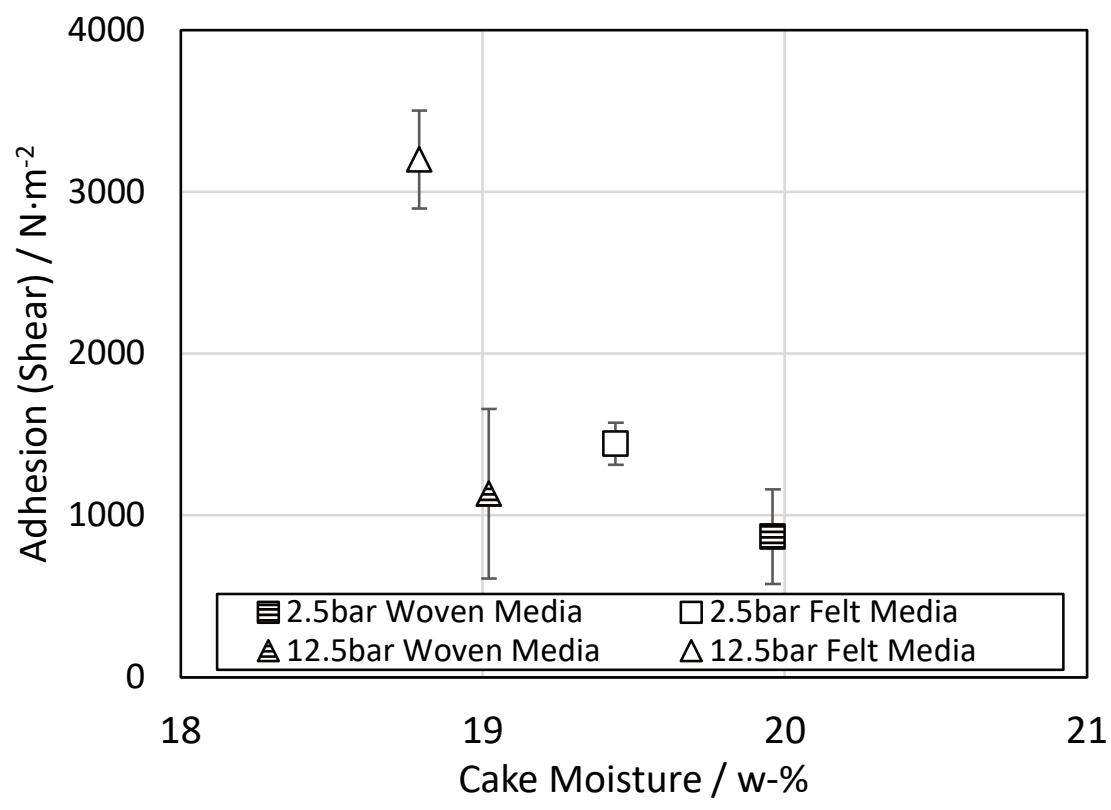

Figure 5 Adhesion (shear) referred to corresponding residual cake moisture 


\section{$5 \quad$ Discussion and conclusion}

Several points are considered in this study. Determination of filter media influences is limited. Wetting behaviour of different fibre materials (corresponding to contact angle of fluid phase) is not discussed since it becomes more important for dehumidified systems. Also, long-term behaviour is not discussed.

The influences of filtration pressure on cake detachment can be summarised. Increasing compaction of the cake leads to higher adhesion and lower cake moistures. Often only the residual moisture of the cake is argued as an influence on the detachment behaviour. However, it could be shown that the adhesion is vice versa and a deeper understanding of the interaction of adhesion and cohesion is necessary.

\section{Acknowledgement}

The authors thank all students and colleagues who have contributed to the successful completion of this work. Special thanks for support go to Thien Sok, Scott Reddick, Dave Hanfland, Paul McCurdie, Todd Wisdom, James Chaponnel, Brent Stokes and Steve Ware from FLSmidth and Christoph Kessler from Karlsruhe Institute of Technology. The regular meetings and critical discussions have contributed significantly to the success of the work.

This work was sponsored by FLSmidth.

\section{References}

Anlauf, H 2019, Wet Cake Filtration: Fundamentals, Equipment, Strategies, Wiley-VCH, Weinheim.

Darcy, H 1856, Les Fontaines Ptrubliques de la Ville de Dijon.

Davies, MP 2011, 'Filtered dry stacked tailings - the fundamentals', Proceedings of the 15th International Conference on Tailings and Mine Waste, Norman B. Keevil Institute of Mining Engineering, Vancouver.

FLSmidth 2020, Sustainability Report 2020, UN Global Compact Office, viewed 9 April 2021, https://ungc-production.s3.us-west2.amazonaws.com/attachments/cop_2021/495014/original/flsmidthsustainabilityreport2020.pdf?1616590187

Fränkle, B, Morsch, P \& Nirschl, H 2021, 'Regeneration assessments of filter fabrics of filter presses in the mining sector', Minerals Engineering, vol. 168, https://doi.org/10.1016/j.mineng.2021.106922

Gomes, RB, De Tomib, G \& Assisa, PS 2016, 'Iron ore tailings dry stacking in Pau Branco mine, Brazil', Journal of Materials Research and Technology, vol. 5, no. 4, pp. 339-344, https://doi.org/10.1016/j.jmrt.2016.03.008

Kozeny, J 1927, 'Ueber kapillare Leitung des Wassers im Boden', Sitzungsber. Akad. Wiss., vol. 136, no. 2a, pp. $271-306$.

Ripperger, S, Gösele, W, Alt, C \& Loewe, T 2013, 'Filtration, 1. fundamentals', Ullmann's Encyclopedia of Industrial Chemistry, Wiley-VCH, Weinheim, https://doi.org/10.1002/14356007.b02_10.pub3

Verein Deutscher Ingenieure 2010, Mechanical Solid-Liquid Separation by Cake Filtration (VDI 2762 Blatt 2), Verein Deutscher Ingenieure, Düsseldorf.

Wakeman, R 2007, 'The influence of particle properties on filtration', Separation and Purification Technology, vol. 58, no. 2, pp. 234-241, https://doi.org/10.1016/j.seppur.2007.03.018

Wang, C, Harbottle, D, Liu, Q \& Xu, Z 2014, 'Current state of fine mineral tailings treatment: A critical review on theory and practice', Minerals Engineering, vol. 58, pp. 113-131, https://doi.org/10.1016/j.mineng.2014.01.018

Weigert, T 2001, Haftung von Filterkuchen bei der Fest-Flüssig-Filtration, VDI-Verlag, Düsseldorf.

Wisdom, T, Neumann, R \& Chaponnel, J 2020, 'Development and testing of the world's largest capacity tailings filter press', in H Quelopana (ed.), Paste 2020: Proceedings of the 23rd International Conference on Paste, Thickened and Filtered Tailings, Gecamin Publications, Santiago, https://doi.org/10.36487/ACG_repo/2052_16 